\title{
Research Article \\ Existence and Uniqueness of Positive Solution for Singular BVPs on Time Scales
}

\author{
Ana Gómez González ${ }^{1}$ and Victoria Otero-Espinar ${ }^{2}$ \\ ${ }^{1}$ Departamento de Matemática Aplicada, Facultade de Matemáticas, Universidade de Santiago de \\ Compostela, 15782 Galicia, Spain \\ ${ }^{2}$ Departamento de Análise Matemática, Facultade de Matemáticas, Universidade de Santiago de \\ Compostela, 15782 Galicia, Spain
}

Correspondence should be addressed to Victoria Otero-Espinar, mvictoria.otero@usc.es

Received 27 March 2009; Accepted 12 May 2009

Recommended by Alberto Cabada

This paper is devoted to derive some sufficient conditions for the existence and uniqueness of positive solutions to a singular second-order dynamic equation with Dirichlet boundary conditions.

Copyright (C) 2009 A. Gómez González and V. Otero-Espinar. This is an open access article distributed under the Creative Commons Attribution License, which permits unrestricted use, distribution, and reproduction in any medium, provided the original work is properly cited.

\section{Introduction}

Hilger [1] introduced the notion of time scale in 1990 in order to unify the theory of continuous and discrete calculus. The field of dynamic equations on time scale contains, links, and extends the classical theory of differential and difference equations, besides many others. There are more time scales than just $\mathbb{R}$ (corresponding to the continuous case) and $\mathbb{N}$ (discrete case) and hence many more classes of dynamic equations.

By time scale we mean a closed subset of the real numbers. Let $\mathbb{T}$ be an arbitrary time scale. We assume that $\mathbb{T}$ has the topology that it inherits from the standard topology on $\mathbb{R}$. Assume that $a<b$ are points in $\mathbb{T}$ and define the time scale interval $[a, b]_{\mathbb{T}}=\{t \in \mathbb{T}: a \leq t \leq$ $b\}$. For $t \in \mathbb{T}$, define the forward jump operator $\sigma: \mathbb{T} \rightarrow \mathbb{T}$ by $\sigma(t)=\inf \{s \in \mathbb{T}: s>t\}$ and the backward jump operator $\rho: \mathbb{T} \rightarrow \mathbb{T}$ by $\rho(t)=\sup \{s \in \mathbb{T}: s<t\}$. In this definition we put $\sigma(t)=t$ if $\mathbb{T}$ attains a maximum $t$ and $\rho(t)=t$ if $\mathbb{T}$ attains a minimum $t$. If $\sigma(t)>t, t$ is said to be right-scattered and if $\sigma(t)=t, t$ is said to be right-dense. If $\rho(t)<t, t$ is said to be left-scattered and if $\rho(t)=t, t$ is said to be left-dense.

A function $f: \mathbb{T} \rightarrow \mathbb{R}$ is said to be rd-continuous provided it is continuous at all rightdense points of $\mathbb{T}$, and its left-sided limit exists at left-dense points of $\mathbb{T}$. For $t \in \mathbb{T}$, the delta 
derivative $f^{\Delta}(t)$ of $f$ at $t$ is defined to be the number (if exists) such that for given $\epsilon>0$, there exists a neighborhood $U$ of $t$ such that

$$
\left|[f(\sigma(t)-f(s))]-f^{\Delta}(t)[\sigma(t)-s]\right| \leq \epsilon|\sigma(t)-s|, \quad \forall s \in U
$$

See [2] for general theory about time scales.

The problem we will consider in this work is of the type

$$
\begin{gathered}
-u^{\Delta \Delta}=g\left(t, u^{\sigma}(t)\right), \\
u(a)=u\left(\sigma^{2}(b)\right)=0 .
\end{gathered}
$$

Under this general form it included the Emden-Fowler equation, which arises in several fields, such as the following:

(i) Astrophysics: related to the stellar structure (gaseous dynamics). In this case the fundamental problem is to investigate the equilibrium configuration of the mass of spherical clouds of gas.

(ii) Gas dynamics and fluid mechanics. The solutions of physical interest in this context are bounded nonoscillatory and possess a positive zero.

(iii) Relativistic mechanics.

(iv) Nuclear physics.

(v) Chemically reacting systems: in the theory of diffusion and reaction this equation appears as governing the concentration $u$ of a substance which disappears by an isothermal reaction at each point of a slab of catalyst.

We refer to Wong [3], for a general historical overview about this equation.

Many works on this equation have been written in the continuous case, and we can cite among others, $[4,5]$ or $[6]$.

On the discrete case we find the book [7] which studies the oscillation properties of the solutions of different difference equations. For the specific problem $u^{\Delta \Delta}(t)+p(t) u^{\gamma}(\sigma(t))=0$, where $p \geq 0$ and $\gamma$ quotient of odd positive numbers, also oscillation properties were studied in $[8]$.

On time scales some results on existence and uniqueness of solutions in the sense of distribution for this equation can be found in the article [9]. Considering classical solutions, oscillation properties have also been studied, in works such as [10] (with delay) or [11].

In the present paper we present some results on time scales considering classical solutions which generalize the ones from the continuous case.

\section{Lower and Upper Solutions Method}

Let $a, b \in \mathbb{T}$, such that $a<\rho(b)$. Let us put $J=\left[a, \sigma^{2}(b)\right]_{\mathbb{T}}, J^{\kappa}=[a, \sigma(b)]_{\mathbb{T}}$, and $\left(J^{\kappa}\right)^{o}=$ $[a, \sigma(b))_{\mathbb{T}}$ if $a \neq \sigma(a)$ and $J=\left(a, \sigma^{2}(b)\right]_{\mathbb{T}}, J^{\kappa}=(a, \sigma(b)]_{\mathbb{T}}$, and $\left(J^{\kappa}\right)^{o}=(a, \sigma(b))_{\mathbb{T}}$ if $a=\sigma(a)$. 
We consider the second-order dynamic equation with Dirichlet boundary conditions,

$$
\begin{gathered}
-u^{\Delta \Delta}(t)=f\left(t, u^{\sigma}(t)\right), \quad t \in\left(J^{\kappa}\right)^{o}, \\
u(a)=A, \quad u\left(\sigma^{2}(b)\right)=B,
\end{gathered}
$$

where $f:\left(J^{\kappa}\right)^{o} \times \mathscr{A} \rightarrow \mathbb{R}, \mathcal{A} \subset \mathbb{R}$, satisfies the following condition.

$\left(\mathrm{H}_{1}\right)$ (i) For every $x \in \mathcal{A}, f(\cdot, x) \in C_{\mathrm{rd}}\left(\left(J^{\kappa}\right)^{o}\right)$,

(ii) $f(t, \cdot)$ is continuous on $\mathcal{A}$ uniformly in $t \in\left(J^{\kappa}\right)^{o}$.

For convenience, we denote

$$
E=\left\{h \in C_{\mathrm{rd}}\left(\left(J^{\kappa}\right)^{o}, \mathbb{R}^{+}\right): \int_{a}^{\sigma(b)}(\sigma(s)-a)\left(\sigma^{2}(b)-s\right) h(s) \Delta s<+\infty\right\} .
$$

We say that $f$ satisfies the condition $\left(\mathrm{H}_{2}\right)$ on $\mathbb{B} \subset\left(J^{\kappa}\right)^{o} \times \mathcal{A}$ if there exists a function $h \in E$ such that

$\left(\mathrm{H}_{2}\right)$

$$
|f(t, x)| \leq h(t), \quad \forall(t, x) \in \mathbb{B}
$$

Definition 2.1. A solution of $(P)$ is a function $u \in C_{\mathrm{rd}}^{2}\left((a, b)_{\mathbb{T}}\right)$ such that $u(t) \in \mathcal{A}$, for all $t \in\left[a, \sigma^{2}(b)\right]_{\mathbb{T}}$, which satisfies the equalities on $(P)$ for each $t \in\left(J^{\kappa}\right)^{o}$, where

$$
C_{\mathrm{rd}}^{2}\left((a, b)_{\mathbb{T}}\right)=\left\{y \in C\left(\left[a, \sigma^{2}(b)\right]_{\mathbb{T}}\right), y^{\Delta \Delta}:\left(J^{\kappa}\right)^{o} \longrightarrow \mathbb{R}, y^{\Delta \Delta} \in C_{\mathrm{rd}}\left((a, b)_{\mathbb{T}}\right)\right\} .
$$

Definition 2.2. We say that $\alpha \in C_{\mathrm{rd}}^{2}\left((a, b)_{\mathbb{T}}\right)$ is a lower solution of $(P)$ if $\alpha(t) \in \mathcal{A}$, for all $t \in\left[a, \sigma^{2}(b)\right]_{\mathbb{T}}$ and

$$
\begin{gathered}
-\alpha^{\Delta \Delta}(t) \leq f\left(t, \alpha^{\sigma}(t)\right), \quad t \in\left(J^{\kappa}\right)^{o}, \\
\alpha(a) \leq A, \quad \alpha\left(\sigma^{2}(b)\right) \leq B .
\end{gathered}
$$

An upper solution $\beta \in C_{\mathrm{rd}}^{2}\left((a, b)_{\mathbb{T}}\right)$ of $(P)$ is defined similarly by reversing the previous inequalities.

We have the following result.

Theorem 2.3. Let $\alpha$ and $\beta$ be, respectively, a lower and upper solution for problem $(P)$, such that $\alpha \leq \beta$ on $\left[a, \sigma^{2}(b)\right]_{\mathbb{T}}$. If $f$ satisfies $\left(\mathrm{H}_{1}\right)$ and the conditions $\left(\mathrm{H}_{2}\right)$ on

$$
\Phi_{\alpha}^{\beta}=\left\{(t, x) \in\left(J^{\kappa}\right)^{o} \times \mathbb{R}: \alpha^{\sigma}(t) \leq x \leq \beta^{\sigma}(t)\right\},
$$

then problem $(P)$ has at least one solution $\tilde{u}$ such that $\alpha \leq \tilde{u} \leq \beta$ on $\left[a, \sigma^{2}(b)\right]_{\mathbb{T}}$. 
Proof. We consider the following modified problem:

$$
\begin{gathered}
-u^{\Delta \Delta}(t)=f^{*}\left(t, u^{\sigma}(t)\right), \quad t \in\left(J^{\kappa}\right)^{o}, \\
u(a)=A, \quad u\left(\sigma^{2}(b)\right)=B .
\end{gathered}
$$

with

$$
f^{*}(t, x)= \begin{cases}f\left(t, \alpha^{\sigma}(t)\right)+\frac{\alpha^{\sigma}(t)-x}{1+\left(\alpha^{\sigma}(t)-x\right)}, & \text { if } x \leq \alpha^{\sigma}(t) \\ f(t, x), & \text { if } \alpha^{\sigma}(t) \leq x \leq \beta^{\sigma}(t) \\ f\left(t, \beta^{\sigma}(t)\right)+\frac{x-\beta^{\sigma}(t)}{1+\left(x-\beta^{\sigma}(t)\right)}, & \text { if } \beta^{\sigma}(t) \leq x\end{cases}
$$

Due to the hypothesis it can be easily checked that $\left(\mathrm{H}_{1}\right)$ and $\left(\mathrm{H}_{2}\right)$ are satisfied by the function $f^{*}$.

Note that, if $u$ is a solution of $\left(P_{m}\right)$ such that $\alpha \leq u \leq \beta$ on $\left[a, \sigma^{2}(b)\right]_{\mathbb{T}}$, then $u$ is a solution of $(P)$, also satisfying $\alpha \leq u \leq \beta$ on $\left[a, \sigma^{2}(b)\right]_{\mathbb{T}}$.

To show that any solution $u$ of $\left(P_{m}\right)$ is between $\alpha$ and $\beta$, let $v(t)=\alpha(t)-u(t)$, and suppose that there exists $t^{*} \in\left[a, \sigma^{2}(b)\right]_{\mathbb{T}}$ such that $v\left(t^{*}\right)>0$. As $v(a) \leq 0$ and $v\left(\sigma^{2}(b)\right) \leq 0$, then there exists $t_{0} \in\left(a, \sigma^{2}(b)\right)_{\mathbb{T}}$ with

$$
v\left(t_{0}\right)=\max \left\{v(t), t \in\left[a, \sigma^{2}(b)\right]_{\mathbb{T}}\right\}>0,
$$

and $v(t)<v\left(t_{0}\right)$ for $t \in\left(t_{0}, \sigma^{2}(b)\right]_{\mathbb{T}}$. The point $t_{0}$ is not simultaneously left-dense and right-scattered (see [12, Theorem 2.1]) (this implies that $\left.(\sigma \circ \rho)\left(t_{0}\right)=t_{0}\right)$, and we have that $v^{\Delta \Delta}\left(\rho\left(t_{0}\right)\right) \leq 0$ (see [12]), so

$$
\begin{aligned}
-v^{\Delta \Delta}\left(\rho\left(t_{0}\right)\right) & =u^{\Delta \Delta}\left(t_{0}\right)-\alpha^{\Delta \Delta}\left(t_{0}\right) \\
& \leq-f^{*}\left(\rho\left(t_{0}\right), u^{\sigma}\left(\rho\left(t_{0}\right)\right)\right)+f\left(\rho\left(t_{0}\right), \alpha^{\sigma}\left(\rho\left(t_{0}\right)\right)\right) \\
& =-\frac{\alpha\left(t_{0}\right)-u\left(t_{0}\right)}{1+\left(\alpha\left(t_{0}\right)-u\left(t_{0}\right)\right)}<0 .
\end{aligned}
$$

So $v^{\Delta \Delta}\left(\rho\left(t_{0}\right)\right)>0$, that is a contradiction. And so we have proved that $v(t) \leq 0, \forall t \in$ $\left[a, \sigma^{2}(b)\right]_{\mathbb{T}}$.

Analogously it can be proved that $u(t) \leq \beta(t), \forall t \in\left[a, \sigma^{2}(b)\right]_{\mathbb{T}}$.

We only need to prove that problem $\left(P_{m}\right)$ has at least one solution.

Consider now the operator $N: C\left(\left[a, \sigma^{2}(b)\right]_{\mathbb{T}}\right) \rightarrow C\left(\left[a, \sigma^{2}(b)\right]_{\mathbb{T}}\right)$, defined by

$$
N u(t)=\phi(t)+\int_{a}^{\sigma(b)} G(t, s) f^{*}\left(s, u^{\sigma}(s)\right) \Delta s
$$


for each $t \in\left[a, \sigma^{2}(b)\right]_{\mathbb{T}}$, where (see [2])

$$
G(t, s)=\frac{1}{\sigma^{2}(b)-a} \begin{cases}(t-a)\left(\sigma^{2}(b)-\sigma(s)\right), & t \leq s, \\ (\sigma(s)-a)\left(\sigma^{2}(b)-t\right), & \sigma(s) \leq t\end{cases}
$$

is Green's function of the problem

$$
\begin{gathered}
-x^{\Delta \Delta}=0, \\
x(a)=x\left(\sigma^{2}(b)\right)=0,
\end{gathered}
$$

and for $t \in\left[a, \sigma^{2}(b)\right]_{\mathbb{T}}$

$$
\phi(t)=A+\frac{B-A}{\sigma^{2}(b)-a}(t-a)
$$

is the solution of $-x^{\Delta \Delta}=0$ such that $\phi(a)=A$ and $\phi\left(\sigma^{2}(b)\right)=B$.

Clearly, $G(t, s)>0$ on $\left(a, \sigma^{2}(b)\right)_{\mathbb{T}} \times\left(a, \sigma^{2}(b)\right)_{\mathbb{T}}, G(t, \cdot)$ is rd-continuous on $[a, \sigma(b)]_{\mathbb{T}}$ and $G(\cdot, s)$ is continuous on $\left[a, \sigma^{2}(b)\right]_{\mathbb{T}}$.

The function $N u$ defined by $(2.9)$ belongs to $C\left(\left[a, \sigma^{2}(b)\right]_{\mathbb{T}}\right)$ because $f^{*}$ checks the conditions $\left(\mathrm{H}_{1}\right)$ and $\left(\mathrm{H}_{2}\right)$ on $\left(J^{\kappa}\right)^{o} \times \mathbb{R}$ and $G(t, s) \leq s(1-s)$, for each $t, s \in\left[a, \sigma^{2}(b)\right]_{\mathbb{T}}$.

It is obvious that $u \in C\left(\left[a, \sigma^{2}(b)\right]_{\mathbb{T}}\right)$ is a solution of $\left(P_{m}\right)$ if and only if $u=N u$. So the problem now is ensuring the existence of fixed-points of $N$.

First of all, $N$ is well defined, is continuous, and $N\left(C\left(\left[a, \sigma^{2}(b)\right]_{\mathbb{T}}\right)\right)$ is a bounded set. The existence of a fixed-point of $N$ follows from the Schauder fixed-point theorem, once we have checked that $N\left(C\left(\left[a, \sigma^{2}(b)\right]_{\mathbb{T}}\right)\right)$ is relatively compact, that using the Ascoli-Arzela theorem is equivalent to proving that $N\left(C\left(\left[a, \sigma^{2}(b)\right]_{\mathbb{T}}\right)\right)$ is an equicontinuous family.

Let $h^{*} \in E$ be the function related to $f^{*}$ by condition $\left(\mathrm{H}_{2}\right)$. We compute the first derivative of $N u$ using [2, Theorem 1.117]

$$
\begin{aligned}
\left|(N u)^{\Delta}(t)\right|= & \frac{1}{\sigma^{2}(b)-a} \mid(B-A)-\int_{a}^{t}(\sigma(s)-a) f^{*}\left(s, u^{\sigma}(s)\right) \Delta s \\
& \quad+\int_{t}^{\sigma(b)}\left(\sigma^{2}(b)-\sigma(s)\right) f^{*}\left(s, u^{\sigma}(s)\right) \Delta s \mid \\
\leq & \frac{1}{\sigma^{2}(b)-a}\left(|B-A|+\int_{a}^{t}(\sigma(s)-a) h^{*}(s) \Delta s+\int_{t}^{\sigma(b)}\left(\sigma^{2}(b)-\sigma(s)\right) h^{*}(s) \Delta s\right) \\
& :=\frac{1}{\sigma^{2}(b)-a}(|B-A|+\lambda(t)) .
\end{aligned}
$$


Finally it is enough to check that $\lambda \in L^{1}\left(\left(J^{\kappa}\right)^{o}\right)$, using integration by parts we obtain

$$
\begin{aligned}
\int_{a}^{\sigma(b)}|\lambda(s)| \Delta s= & \int_{a}^{\sigma(b)} \lambda(s) \Delta s \\
= & \lim _{r \rightarrow \sigma(b)} \int_{a}^{r}\left(\int_{a}^{t}(\sigma(s)-a) h^{*}(s) \Delta s\right) \Delta t \\
& +\lim _{r \rightarrow a^{+}} \int_{r}^{\sigma(b)}\left(\int_{t}^{\sigma(b)}\left(\sigma^{2}(b)-\sigma(s)\right) h^{*}(s) \Delta s\right) \Delta t \\
= & 2 \int_{a}^{\sigma(b)}(\sigma(s)-a)\left(\sigma^{2}(b)-\sigma(s)\right) h^{*}(s) \Delta s \\
& -\lim _{r \rightarrow \sigma(b)^{-}}\left(\sigma^{2}(b)-r\right) \int_{a}^{r}(\sigma(s)-a) h^{*}(s) \Delta s \\
& -\lim _{r \rightarrow a^{+}}(r-a) \int_{r}^{\sigma(b)}\left(\sigma^{2}(b)-\sigma(s)\right) h^{*}(s) \Delta s<+\infty,
\end{aligned}
$$

due to $h^{*} \in E$, and the fact

$$
\begin{gathered}
\left(\sigma^{2}(b)-r\right) \int_{a}^{r}(\sigma(s)-a) h^{*}(s) \Delta s \leq \int_{a}^{\sigma(b)}(\sigma(s)-a)\left(\sigma^{2}(b)-\sigma(s)\right) h^{*}(s) \Delta s, \\
(r-a) \int_{r}^{\sigma(b)}\left(\sigma^{2}(b)-\sigma(s)\right) h^{*}(s) \Delta s \leq \int_{a}^{\sigma(b)}(\sigma(s)-a)\left(\sigma^{2}(b)-\sigma(s)\right) h^{*}(s) \Delta s .
\end{gathered}
$$

And so the result is proved.

\section{Existence and Uniqueness of Positive Solution}

Let $g:\left(J^{\kappa}\right)^{o} \times(0,+\infty) \rightarrow \mathbb{R}$ in the condition $\left(\mathrm{H}_{1}\right)$, where $\mathcal{A}=(0,+\infty)$, and consider the problem

$$
\begin{gathered}
-u^{\Delta \Delta}(t)=g\left(t, u^{\sigma}(t)\right), \quad t \in\left(J^{\kappa}\right)^{o}, \\
u(a)=u\left(\sigma^{2}(b)\right)=0 .
\end{gathered}
$$

We will deduce the existence of solution to $(Q)$ by supposing that the following hypothesis holds.

$\left(\mathrm{H}_{3}\right)$ There exists a constant $L>0$ such that for any compact set $D \subset\left(J^{\kappa}\right)^{o}$, there is $\varepsilon=\varepsilon_{D}>0$ :

$$
g(t, x)>L, \quad \forall(t, x) \in D \times(0, \varepsilon] .
$$


Theorem 3.1. Suppose that $\left(\mathrm{H}_{1}\right)$ and $\left(\mathrm{H}_{3}\right)$ hold. If, for any $\delta>0$, $g$ satisfies the condition $\left(\mathrm{H}_{2}\right)$ on $\left(J^{\kappa}\right)^{o} \times[\delta,+\infty)$, then problem $(Q)$ has at least one solution.

Proof. Let's consider $\left\{a_{n}\right\}_{n \geq 1},\left\{b_{n}\right\}_{n \geq 1} \subset\left(J^{\kappa}\right)^{o}$ as two sequences such that $\left\{a_{n}\right\}_{n \geq 1} \subset$ $(a,(a+\sigma(b)) / 2)_{\mathbb{T}}$ is strictly decreasing to $a$ if $a=\sigma(a)$, and $a_{n}=a$ for all $n \geq 1$ if $a<\sigma(a)$, and $\left\{b_{n}\right\}_{n \geq 1} \subset((a+\sigma(b)) / 2, \sigma(b))_{\mathbb{T}}$ is strictly increasing to $\sigma(b)$ if $\rho(\sigma(b))=\sigma(b), b_{n}=\rho(\sigma(b))$ for all $n \geq 1$ if $\rho(\sigma(b))<\sigma(b)$. We denote as $D_{n}:=\left[a_{n}, b_{n}\right]_{\mathbb{T}} \subset\left(J^{\kappa}\right)^{o}, n \geq 1$.

Due to the first hypothesis, we can then ensure the existence of $\varepsilon_{n}>0$ such that $g(t, x)>L$, for all $(t, x) \in D_{n} \times\left(0, \varepsilon_{n}\right]$. We can suppose, without restriction that $\left\{\varepsilon_{n}\right\}$ is a decreasing sequence and $\lim _{n \rightarrow+\infty} \varepsilon_{n}=0$.

Consider the function $\gamma: \quad\left[a, a_{1}\right]_{\mathbb{T}} \rightarrow \mathbb{R}^{+}$, such that $\gamma(a)=0$, and if $a \neq a_{1}$ then $\gamma(t)=$ $\varepsilon_{2}$ for $t \in\left[a_{2}, a_{1}\right], \gamma(t)=\varepsilon_{n}$, for all $t \in D_{n} \backslash D_{n-1}$, with $n \geq 3$. Since $\gamma$ is nondecreasing, we obtain that for $t \in\left[a, a_{1}\right]$

$$
\gamma_{1}(t):=\frac{1}{\sigma^{2}(b)-a} \int_{a}^{t} \gamma(s) \Delta s \leq \gamma(t)
$$

is continuous and increasing. Repeating this argument twice, for $i=2,3$ we define

$$
\gamma_{i}(t):=\frac{1}{\sigma^{2}(b)-a} \int_{a}^{t} \gamma_{i-1}(s) \Delta s \leq \gamma(t)
$$

So $\gamma_{3} \in C^{2}\left(\left[a, a_{1}\right]_{\mathbb{T}}\right)$ is a convex function verifying $\gamma_{3}(t) \leq \varepsilon_{n}, \forall t \in D_{n} \backslash D_{n-1}, n \geq 2$.

Analogously, we can consider $\tilde{\gamma}:\left[b_{1}, \sigma^{2}(b)\right]_{\mathbb{T}} \rightarrow \mathbb{R}^{+}$, such that $\tilde{\gamma}\left(\sigma^{2}(b)\right)=0, \tilde{\gamma}(\sigma(b))=$ $0, \tilde{\gamma}(t)=\varepsilon_{2}$, for all $t \in\left[b_{1}, b_{2}\right]$ and $\tilde{\gamma}(t)=\varepsilon_{n}$, for all $t \in D_{n} \backslash D_{n-1}$, with $n \geq 3$. Taking $\tilde{\gamma}_{0}=\tilde{\gamma}$, we obtain, for $i=1,2,3$, that for $t \in\left[b_{1}, \sigma^{2}(b)\right]$

$$
\tilde{\gamma}_{i}(t):=\frac{1}{\sigma^{2}(b)-a} \int_{t}^{\sigma^{2}(b)} \tilde{\gamma}_{i-1}(s) \Delta s \leq \tilde{\gamma}(t)
$$

is continuous and decreasing, and $\tilde{\gamma}_{3} \in C^{2}\left(\left[b_{1}, \sigma^{2}(b)\right]_{\mathbb{T}}\right)$ a convex function such that $\tilde{\gamma}_{3}(t) \leq$ $\varepsilon_{n}, \forall t \in D_{n} \backslash D_{n-1}, n \geq 2$.

We now define

$$
\alpha(t)= \begin{cases}r_{3}(t), & t \in\left[a, a_{1}\right]_{\mathbb{T}}, \\ \alpha^{*}(t), & t \in\left[a_{1}, b_{1}\right]_{\mathbb{T}}, \\ \widetilde{\gamma}_{3}(t), & t \in\left[b_{1}, \sigma^{2}(b)\right]_{\mathbb{T}},\end{cases}
$$

with $\alpha^{*}(t)$ a convenient function, so that $\alpha \in C^{2}\left(\left[a, \sigma^{2}(b)\right]_{\mathbb{T}}\right)$, and $0<\alpha(t) \leq \varepsilon_{1}$ for all $t \in$ $\left[a_{1}, b_{1}\right]_{\mathbb{T}}$.

So $\alpha \in C^{2}\left(\left[a, \sigma^{2}(b)\right]_{\mathbb{T}}\right)$ is a function such that $\alpha(a)=\alpha\left(\sigma^{2}(b)\right)=0, \alpha(t)>0$ for $t \in$ $(a, \sigma(b))_{\mathbb{T}}, \alpha(t) \leq \varepsilon_{1}$ for $t \in D_{1}$, and $\alpha(t) \leq \varepsilon_{n}$, for $t \in D_{n} \backslash D_{n-1}, n \geq 2$.

In this way, we note that

$$
g(t, x)>L, \quad \forall(t, x) \in\left(J^{\mathcal{K}}\right)^{o} \times\{x \in(0,+\infty): 0<x \leq \alpha(t)\} .
$$


Let $m_{0}=\min \left\{1, L /\left(\|\alpha\|^{\Delta \Delta}+1\right)\right\}$.

Let $F(t, x) \geq g(t, x)$, for all $(t, x) \in\left(J^{\mathcal{K}}\right)^{o} \times(0,+\infty)$, with $F$ in the conditions $\left(\mathrm{H}_{1}\right)$. We will prove that if $v \in C_{\mathrm{rd}}^{2}\left(\left(a, \sigma^{2}(b)\right)_{\mathbb{T}}\right)$ is any solution of

$$
-v^{\Delta \Delta}=F\left(t, v^{\sigma}(t)\right)
$$

with $v(a) \geq 0, v\left(\sigma^{2}(b)\right) \geq 0$ and $v(t)>0$ for all $t \in\left(J^{\kappa}\right)^{o}$, then,

$$
v(t) \geq m_{0} \alpha(t), \quad \forall t \in\left[a, \sigma^{2}(b)\right]_{\mathbb{T}}
$$

Suppose there exists $t \in\left[a, \sigma^{2}(b)\right]_{\mathbb{T}}$, such that $v(t)-m_{0} \alpha(t)<0$. Then, we can assure, using arguments analogous to the ones in the proof of Theorem 2.3 , that there exists $t^{*} \in\left(a, \sigma^{2}(b)\right)_{\mathbb{T}}$ verifying

$$
v^{\Delta \Delta}\left(\rho\left(t^{*}\right)\right) \geq m_{0} \alpha^{\Delta \Delta}\left(\rho\left(t^{*}\right)\right)
$$

which implies that

$$
L<F\left(\rho\left(t^{*}\right), v^{\sigma}\left(\rho\left(t^{*}\right)\right)\right)=-v^{\Delta \Delta}\left(\rho\left(t^{*}\right)\right) \leq-m_{0} \alpha^{\Delta \Delta}\left(\rho\left(t^{*}\right)\right)<m_{0}\left(\left\|\alpha^{\Delta \Delta}\right\|+1\right) \leq L
$$

which is a contradiction.

We define now, for each $n \in \mathbb{N}$

$$
\tilde{g}_{n}(t, x):=\max \left\{g\left(\tau_{n}(t), x\right), g(t, x)\right\}
$$

where, for each $t \in\left(J^{\mathcal{K}}\right)^{o}$

$$
\tau_{n}(t)=\max \left\{a_{n}, \min \left\{t, b_{n}\right\}\right\}
$$

For each $n$ we have that $\tilde{g}_{n}$ is a function verifying $\left(\mathrm{H}_{1}\right)$ on $\left(J^{\mathcal{K}}\right)^{o} \times(0,+\infty)$, and $\tilde{g}_{n}(t, x) \geq g(t, x)$, for all $(t, x) \in J \times(0,+\infty)$ and $\tilde{g}_{n}(t, x)=g(t, x)$, for $t \in D_{n}$.

The sequence $\left\{\tilde{g}_{n}\right\}_{n}$ converges to $g$ uniformly in every set of the form $D \times(0,+\infty)$, where $D \subset\left(J^{\kappa}\right)^{o}$ is a compact set.

Defining, by induction, $g_{1}(t, x)=\tilde{g}_{1}(t, x)$ and for $n>1$

$$
g_{n+1}(t, x)=\min \left\{g_{n}(t, x), \widetilde{g}_{n+1}(t, x)\right\}
$$

we have that, for each $n$, the function $g_{n}$ satisfies the condition $\left(\mathrm{H}_{1}\right)$ on $\left(J^{\kappa}\right)^{o} \times(0,+\infty)$. As well $g_{1} \geq g_{2} \geq \cdots \geq g_{n} \geq \cdots \geq g$, and $\left\{g_{n}\right\}_{n}$ converges to $g$ uniformly, in every set of the form $D \times(0,+\infty)$, where $D \subset\left(J^{\kappa}\right)^{o}$ is a compact set.

It is also verified that $g_{n}(t, x)=g(t, x), t \in\left[a_{n}, b_{n}\right], x \in(0,+\infty)$. 
Now we define the following problems:

$$
\begin{gathered}
u^{\Delta \Delta}(t)+g_{n}\left(t, u^{\sigma}(t)\right)=0, \quad t \in\left(J^{\kappa}\right)^{o} \\
u(a)=u\left(\sigma^{2}(b)\right)=\varepsilon_{n} .
\end{gathered}
$$

We will prove that for any $c \in\left(0, \varepsilon_{n}\right]$, the constant function $\alpha_{n} \equiv c$ is a (strict) lower solution for $(Q)_{n}$.

It is obvious that $c=\alpha_{n}(a) \leq \varepsilon_{n}$, and $c=\alpha_{n}\left(\sigma^{2}(b)\right) \leq \varepsilon_{n}$. Now we have to prove that

$$
g_{n}(t, c)>0, \quad t \in\left(J^{\mathcal{K}}\right)^{o}, c \in\left(0, \varepsilon_{n}\right]
$$

For $n=1$, let $c \in\left(0, \varepsilon_{1}\right]$ such that

$$
g_{1}(t, c)=\widetilde{g}_{1}(t, c)=\max \left\{g\left(\tau_{1}(t), c\right), g(t, c)\right\} \geq g\left(\tau_{1}(t), c\right)>L
$$

Suppose now that $g_{n}(t, c)>0, t \in\left(J^{\kappa}\right)^{o}, c \in\left(0, \varepsilon_{n}\right]$, for a given $n \geq 1$, and we will check that $g_{n+1}(t, c)>0, t \in\left(J^{\mathcal{K}}\right)^{o}$. Let $c \in\left(0, \varepsilon_{n+1}\right]$ such that

$$
g_{n+1}(t, c)=\min \left\{g_{n}(t, c), \tilde{g}_{n+1}(t, c)\right\} \geq \min \left\{g_{n}(t, c), g\left(\tau_{n+1}(t), c\right)\right\}>0
$$

Thus the assertion is proved.

Moreover, as $g_{n} \geq g_{n+1}$ on $\left(J^{\kappa}\right)^{o} \times(0,+\infty)$, it can be easily checked that any solution $u_{n}$ of $(Q)_{n}$ is an upper solution for $(Q)_{n+1}$.

To show that problem $(Q)_{1}$ has at least one solution. We fix a constant $M \geq \varepsilon_{1}$. From the assumption imposed, there exists a function $h_{M} \in C_{\mathrm{rd}}\left(\left(J^{\kappa}\right)^{o}, \mathbb{R}^{+}\right)$such that

$$
|g(t, x)| \leq h_{M}(t), \quad \forall t \in\left(J^{\kappa}\right)^{o}, x \geq M
$$

Note that

$$
\left|g\left(\tau_{1}(t), x\right)\right| \leq h_{M}\left(\tau_{1}(t)\right) \leq R, \quad \forall t \in\left(J^{\kappa}\right)^{o}, \forall x \geq M,
$$

with $R>0$ is a suitable constant.

Set $q(t):=h_{M}(t)+R$. We have that $q \in C_{\mathrm{rd}}\left(\left(J^{\kappa}\right)^{o},(0,+\infty)\right)$, and

$$
\left|g_{1}(t, x)\right| \leq q(t), \quad \forall t \in J, x \geq M
$$

Let $\beta \in C_{\mathrm{rd}}^{2}\left((a, b)_{\mathbb{T}}\right)$ be a solution of the boundary value problem:

$$
\begin{gathered}
u^{\Delta \Delta}(t)+q(t)=0, \\
u(a)=u\left(\sigma^{2}(b)\right)=M .
\end{gathered}
$$


It is easy to check that such a function exists and that $\beta(t) \geq M$, for all $t \in\left[a, \sigma^{2}(b)\right]_{\mathbb{T}}$, and thus

$$
\beta^{\Delta \Delta}(t)+g_{1}\left(t, \beta^{\sigma}(t)\right)=-q(t)+g_{1}\left(t, \beta^{\sigma}(t)\right) \leq 0,
$$

which implies that $\beta$ is an upper solution of $(Q)_{1}$.

Taking $\alpha_{1} \equiv \varepsilon_{1}$, we have that $\alpha_{1}$ and $\beta_{1}:=\beta$ are, respectively, a lower and upper solutions of $(Q)_{1}$. Moreover $\alpha_{1}(t) \leq \beta_{1}(t)$, for all $t \in\left[a, \sigma^{2}(b)\right]_{\mathbb{T}}$. Then, by Theorem 2.3 there exists a solution $u_{1}$ of $(Q)_{1}$ such that

$$
\varepsilon_{1}=\alpha_{1}(t) \leq u_{1}(t) \leq \beta_{1}(t), \quad \forall t \in\left[a, \sigma^{2}(b)\right]_{\mathbb{T}} .
$$

Proceeding by induction, using $\alpha_{n} \equiv \varepsilon_{n}, \beta_{n} \equiv u_{n-1}$, lower and upper solution of $(Q)_{n}, n \geq 2$, we obtain (via Theorem 2.3) a sequence $\left\{u_{n}\right\}$ of solutions of $(Q)_{n}$, such that $\varepsilon_{n} \leq u_{n}(t) \leq$ $u_{n-1}(t)$, for all $t \in\left[a, \sigma^{2}(b)\right]_{\mathbb{T}}$ and $u_{n}(a)=u_{n}\left(\sigma^{2}(b)\right)=\varepsilon_{n}, n \geq 2$.

Since $g_{n} \geq g$ on $\left(J^{\kappa}\right)^{o} \times(0,+\infty)$, and $u_{n}$ is a solution of $(Q)_{n}$ with $u_{n}(t)>0$ for all $t \in\left(J^{\kappa}\right)^{o}$, then $u_{n}(t) \geq m_{0} \alpha(t)$, for every $t \in\left[a, \sigma^{2}(b)\right]_{\mathbb{T}}$.

To end, we will prove that

$$
\tilde{u}(t):=\lim _{n \rightarrow+\infty} u_{n}(t)
$$

is a solution of problem $(Q)$.

We have that, for any $n \geq 1$,

$$
m_{0} \alpha(t) \leq \tilde{u}(t) \leq u_{n}(t), \quad \forall t \in\left[a, \sigma^{2}(b)\right]_{\mathbb{T}} .
$$

Now let $D \subset\left(J^{\kappa}\right)^{o}$ be a compact interval. There exists an index $n^{*}=n^{*}(D) \in \mathbb{N}$ such that $D \subset D_{n}$ for all $n \geq n^{*}$. Let $n \geq n^{*}$, if $t \in D$, then

$$
0=u_{n}^{\Delta \Delta}(t)+g_{n}\left(t, u_{n}^{\sigma}(t)\right)=u_{n}^{\Delta \Delta}(t)+g\left(t, u_{n}^{\sigma}(t)\right) .
$$

Hence, $u_{n}$ verifies the first equality of problem $(Q)$ for all $t \in D$, and for all $n \geq n^{*}$. Moreover

$$
\sup \left\{|g(t, x)|, t \in D, m_{0} \alpha(t) \leq x \leq u_{n^{*}}(t)\right\}<+\infty .
$$

Then by the Ascoli-Arzelá theorem, we can conclude that $\tilde{u}$ is a solution of $(Q)$ in $D$. Since the compact $D$ was arbitrary, we have that $\tilde{u} \in C\left((a, \sigma(b))_{\mathbb{T}}\right)$, and

$$
\tilde{u}^{\Delta \Delta}+g\left(t, \tilde{u}^{\sigma}(t)\right)=0, \quad \forall t \in\left(J^{\kappa}\right)^{o} .
$$

As $\tilde{u}(a)=\tilde{u}\left(\sigma^{2}(b)\right)=\lim _{n \rightarrow+\infty} \varepsilon_{n}=0$, we just have to check the continuity of $\tilde{u}$ in $a$ and $\sigma^{2}(b)$. 
Let $\varepsilon>0$. Take $n_{\varepsilon}$ in such a way that $u_{n_{\varepsilon}}(a)<\varepsilon$. From the continuity of $u_{n_{\varepsilon}}(t)$ at $a$, it follows that we can find a constant $\delta=\delta_{\varepsilon}>0$ such that

$$
0<u_{n_{\varepsilon}}(t)<\varepsilon, \quad \forall t \in(a, a+\delta)_{\mathbb{T}} .
$$

So

$$
0<m_{0} \alpha(t) \leq \tilde{u}(t) \leq u_{n_{\varepsilon}}(t)<\varepsilon, \quad \forall t \in(a, a+\delta)_{\mathbb{T}} .
$$

Using the same argument the continuity at $\sigma^{2}(b)$ is proved.

In order to prove the uniqueness of the solution to problem $(Q)$ we have the following result.

Theorem 3.2. Under the hypotheses of Theorem 3.1, if $g(t, \cdot)$ is strictly decreasing in $x$, for each $t \in\left(J^{\kappa}\right)^{o}$, then problem $(Q)$ has a unique solution.

Proof. By Theorem 3.1 the problem $(Q)$ has at least one solution.

Suppose that there exist $v_{1}$ and $v_{2}$, solutions of $(Q)$ and $t^{*} \in\left(J^{\kappa}\right)^{o}$ such that $v_{1}\left(t^{*}\right)>$ $v_{2}\left(t^{*}\right)$. If this occurs, setting $z(t):=v_{1}(t)-v_{2}(t)$ we can find $t_{0} \in\left(a, \sigma^{2}(b)\right)_{\mathbb{T}}$ such that $z\left(t_{0}\right)>0$ and $z^{\Delta \Delta}\left(\rho\left(t_{0}\right)\right) \leq 0$. Using the decreasing property of $g(t, x)$ in $x$, we obtain

$$
-z^{\Delta \Delta}\left(\rho\left(t_{0}\right)\right)=g\left(\rho\left(t_{0}\right), v_{1}^{\sigma}\left(\rho\left(t_{0}\right)\right)\right)-g\left(\rho\left(t_{0}\right), v_{2}^{\sigma}\left(\rho\left(t_{0}\right)\right)\right)<0,
$$

that is a contradiction.

Corollary 3.3. Let $p_{1}, p_{2} \in C_{\mathrm{rd}}\left(\left(J^{\kappa}\right)^{o}\right)$, let $p_{1}>0$ on $\left(J^{\kappa}\right)^{o}$ and $p_{1}+\left|p_{2}\right| \in E$. If $\psi \in C((0,+\infty)), \psi>$ 0 is strictly decreasing on $(0,+\infty)$ and $\lim _{x \rightarrow 0^{+}} \psi(x)=+\infty$, then problem

$$
\begin{gathered}
u^{\Delta \Delta}+p_{1}(t) \psi(t)=p_{2}(t), \\
u(a)=u\left(\sigma^{2}(b)\right)=0
\end{gathered}
$$

has a unique solution in $C_{\mathrm{rd}}^{2}(J)$.

Proof. Setting $g(t, x):=p_{1}(t) \psi(x)-p_{2}(t)$, and for each $\delta>0, h_{\delta}(t):=p_{1}(t) \psi(\delta)+\left|p_{2}(t)\right|$, it is clear that the hypothesis in Theorem 3.2 holds. So, applying the result we have ensured the existence of one unique solution to the problem.

Example 3.4. Let $\mathbb{T}=C$ be the classical ternary Cantor set, and $a=0, b=1$. For every $t \in \mathbb{T}$, let $I_{t}=\left\{i \in I: t_{i} \in R \cap[0, t)\right\}$ with $R=\left\{t_{i}\right\}_{i \in I}$ the set of all right-scattered points of $\mathbb{T}$. 
If we take $p_{1}(t)=1 / t^{1 / 2} \sigma(t)(1-t), p_{2}(t)=0$ and

$$
\psi(x)= \begin{cases}\frac{1}{2}\left(\frac{1}{x^{2}}-\sum_{k \in I_{x}} \mu\left(t_{k}\right)^{2}\right) & \text { if } x \in \mathbb{T}, x \neq 0 \\ \frac{1}{t_{i} x+t_{i}^{2}}-\frac{1}{2} \sum_{k \in I_{t_{i}}} \mu\left(t_{k}\right)^{2} & \text { if } x \in\left(t_{i}, \sigma\left(t_{i}\right)\right) \\ \frac{1}{5 e} e^{1 / x} & \text { if } x \geq 1\end{cases}
$$

then problem (3.31) has a unique solution.

\section{Acknowledgments}

This research is partially supported by D.G.I. and F.E.D.E.R. project MTM2007-61724 and by the Xunta of Galicia and F.E.D.E.R. project PGIDIT06PXIB207023PR, Spain.

\section{References}

[1] S. Hilger, "Analysis on measure chains-a unified approach to continuous and discrete calculus," Results in Mathematics, vol. 18, no. 1-2, pp. 18-56, 1990.

[2] M. Bohner and A. Peterson, Dynamic Equations on Time Scales: An Introduction with Applications, Birkhäuser, Boston, Mass, USA, 2001.

[3] J. S. W. Wong, “On the generalized Emden-Fowler equation,” SIAM Review, vol. 17, pp. 339-360, 1975.

[4] R. P. Agarwal, D. O’Regan, V. Lakshmikantham, and S. Leela, "An upper and lower solution theory for singular Emden-Fowler equations," Nonlinear Analysis: Real World Applications, vol. 3, no. 2, pp. 275-291, 2002.

[5] P. Habets and F. Zanolin, "Upper and lower solutions for a generalized Emden-Fowler equation," Journal of Mathematical Analysis and Applications, vol. 181, no. 3, pp. 684-700, 1994.

[6] Y. Zhang, "Positive solutions of singular sublinear Emden-Fowler boundary value problems," Journal of Mathematical Analysis and Applications, vol. 185, no. 1, pp. 215-222, 1994.

[7] R. P. Agarwal, M. Bohner, S. R. Grace, and D. O'Regan, Discrete Oscillation Theory, Hindawi, New York, NY, USA, 2005.

[8] E. Akin-Bohner and J. Hoffacker, "Oscillation properties of an Emden-Fowler type equation on discrete time scales," Journal of Difference Equations and Applications, vol. 9, no. 6, pp. 603-612, 2003.

[9] R. P. Agarwal, V. Otero-Espinar, K. Perera, and D. R. Vivero, "Multiple positive solutions in the sense of distributions of singular BVPs on time scales and an application to Emden-Fowler equations," Advances in Difference Equations, vol. 2008, Article ID 796851, 13 pages, 2008.

[10] Z. Han, S. Sun, and B. Shi, "Oscillation criteria for a class of second-order Emden-Fowler delay dynamic equations on time scales," Journal of Mathematical Analysis and Applications, vol. 334, no. 2, pp. 847-858, 2007.

[11] M. Bohner, L. Erbe, and A. Peterson, "Oscillation for nonlinear second order dynamic equations on a time scale," Journal of Mathematical Analysis and Applications, vol. 301, no. 2, pp. 491-507, 2005.

[12] R. A. Khan, J. J. Nieto, and V. Otero-Espinar, "Existence and approximation of solution of three-point boundary value problems on time scales," Journal of Difference Equations and Applications, vol. 14, no. 7, pp. 723-736, 2008. 